\title{
Human Metabolism of the Anabolic Steroid Methasterone: Detection and Kinetic Excretion of New Phase I Urinary Metabolites and Investigation of Phase II Metabolism by GC-MS and UPLC-MS/MS
}

\author{
Wendell S. Magalhães, ${ }^{\circledR *, a}$ Bruno C. Garrido, ${ }^{b}$ Gustavo A. Cavalcanti, ${ }^{a}$ \\ Monica C. Padilha, ${ }^{a}$ Alessandro Casilli, ${ }^{a}$ Henrique M. G. Pereira ${ }^{a}$ and \\ Francisco R. de Aquino Neto*,a \\ ${ }^{a}$ Laboratório Brasileiro de Controle de Dopagem, Instituto de Química, \\ Universidade Federal do Rio de Janeiro, 21941-598 Rio de Janeiro-RJ, Brazil \\ ${ }^{b}$ Divisão de Metrologia Química, Instituto Nacional de Metrologia, \\ Qualidade e Tecnologia, 25250-020 Duque de Caxias-RJ, Brazil
}

\begin{abstract}
Methasterone is a designer anabolic steroid that is prohibited for athletes and is monitored by anti-doping laboratories. In this work, our objective is to discover new human phase I metabolites, define their excretion kinetics for 30 days and analyze their phase II metabolism (sulfate, cysteine and $\mathrm{N}$-acetylcysteine conjugates). Urine samples from four volunteers were analyzed by chromatographic techniques. Through gas chromatography coupled to mass spectrometry analysis it was possible to detect methasterone and its nine phase I metabolites in the urine samples after glucuronide enzymatic hydrolysis, from which one were previously unreported. These nine compounds were not excreted in free form. The new proposed metabolite is $17 \beta$-hydroxy$2 \alpha, 17 \alpha$-dimethyl-5 $\beta$-androstan-3-one, obtained from the epimerization at $\mathrm{C} 5$. The $3 \alpha$-hydroxy metabolite, currently monitored by anti-doping laboratories, was the most abundant and was detected for the longest time. Furthermore, four other long-term metabolites were identified. By ultra-performance liquid chromatography coupled to tandem mass spectrometry, only the drug and a known metabolite were detected after glucuronide hydrolysis, and phase II metabolites were not found. Thus, our results contribute to elucidating methasterone metabolism, including long-term metabolites besides of the $3 \alpha$-hydroxy in routine doping analysis, which is very important due to variation in human metabolism.
\end{abstract}

Keywords: methasterone, doping, human metabolism, gas chromatography, ultra-performance liquid chromatography

\section{Introduction}

Anabolic androgenic steroids are testosterone analogs that increase muscle mass due to the anabolic effect. Consequently, they improve the performance of athletes and have been used since $1950 .{ }^{1}$ Scientific investigations aimed at the detection of anabolic steroid abuse in sports began towards the end of the 1960s, ${ }^{2}$ and the use of such substances by athletes began to be monitored during the Montreal Olympic Games in 1976. Since the routine analyses used in doping control are based on previous knowledge of the target analytes, some steroids are designed to evade anti-doping detection. Thus, they have been named

*e-mail: radler@iq.ufrj.br, magalhaes.wendell@gmail.com as "designer steroids". ${ }^{3}$ Due to the limited or even absent information about the metabolism and toxicity of such drugs, they might pose an extremely high risk to the health of their users. A known designer steroid is methasterone (17 $\beta$-hydroxy-2 $\alpha, 17 \alpha$-dimethyl-5 $\alpha$-androstan-3-one, Figure 1), also called 17-methyldrostanolone, which was included in the World Anti-Doping Agency (WADA) Prohibited List in $2006^{4}$ and is prohibited as other steroids. ${ }^{5}$ This drug has already been reported to cause serious liver damage in some subjects. ${ }^{6,7}$

Steroids are normally excreted in urine in their original form for only a limited period of time. This is the reason why the discovery of metabolites that can widen the detection time window is very important for doping analysis. Steroid metabolites are generated by metabolic reactions, classified 


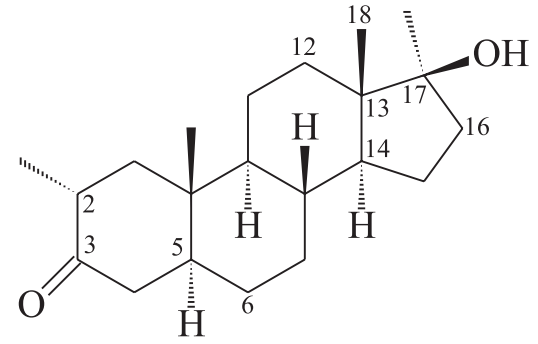

Figure 1. Chemical structure of methasterone (17 $\beta$-hydroxy$2 \alpha, 17 \alpha$-dimethyl-5 $\alpha$-androstan-3-one), emphasizing the identification of important carbon atoms for the metabolism study.

as phase I (e.g., oxidation, reduction and hydrolysis) and phase II (conjugation) before being excreted in urine. ${ }^{8}$ The expected phase I metabolism routes for $17 \alpha$-methyl$17 \beta$-hydroxy- $5 \alpha$-androstane steroids as methasterone are $\mathrm{C} 3$-reduction, $\mathrm{C} 17$-epimerization, oxidation at $\mathrm{C} 6, \mathrm{C} 16$ and/or $\mathrm{C} 18$ and $\mathrm{C} 18$-dehydration, leading to the loss of the methyl group at $\mathrm{C} 18 .^{9-12}$ Evidence regarding these metabolic routes has been reported in several papers using in vitro and in vivo methods, which have detected oxidation at additional positions, such as at $\mathrm{C} 2, \mathrm{C} 12$ and methyl groups. ${ }^{13-17}$ Furthermore, glucuronide and sulfate conjugates of methasterone and its phase I metabolites were detected in human urine ${ }^{17,18}$ and in experiments using equine enzymes in vitro. ${ }^{19}$ These publications studied the human metabolism of methasterone using a limited number of volunteers, reported few sulfate metabolites and did not evaluate the excretion of cysteine (Cys) and/or $\mathrm{N}$-acetylcysteine (NAC) conjugates. Therefore, the goals of the present work are to find new methasterone phase I and phase II human metabolites using a greater number of volunteers and establishing their excretion kinetics, including long-term metabolites besides of the $3 \alpha$-hydroxy metabolite that is already monitored in routine anti-doping analysis, because variations in human metabolism can generate metabolites that are not currently monitored. For this, the chromatographic techniques of gas chromatography coupled to mass spectrometry (GC-MS) and ultra-performance liquid chromatography coupled to tandem mass spectrometry (UPLC-MS/MS) were used.

\section{Experimental}

\section{Chemicals and reagents}

The following chemicals and reagents were used: tert-butylmethylether (TBME), methanol, acetonitrile $(\mathrm{ACN})$ and formic acid, all of pesticide grade from Tedia (Farfield, Ohio, USA). $N$-Methyl- $N$-(trimethylsilyl)trifluoroacetamide (MSTFA) from Chem Fabrik Karl Bucher-Gmbh (Waldstetten, Germany). Ammonium iodide
$\left(\mathrm{NH}_{4} \mathrm{I}\right), 2$-mercaptoethanol and $\beta$-glucuronidase from Escherichia coli (E. coli) from Sigma (St. Louis, MO, USA). Potassium bicarbonate from Spectrum (New Brunswick, New Jersey, USA). Di-sodium hydrogen phosphate, sodium dihydrogen phosphate and potassium carbonate from Merck KGaA (Darmstadt, Germany). 17 $\alpha$-Methyltestosterone (internal standard, IS), methasterone, $5 \alpha$-androstadione and $5 \beta$-androstadione standards from Steraloids (Newport, Rhode Island, USA). Stock solutions of the steroid standards were prepared in methanol at a concentration of $1.0 \mathrm{mg} \mathrm{mL}^{-1}$ and were further diluted to the appropriate working solutions, which were sealed and kept at $-20{ }^{\circ} \mathrm{C}$ until use.

\section{"Nutritional supplement” M-Drol}

A bottle of the "nutritional supplement" M-Drol, produced by Competitive Edge labs, was purchased from a sports supplement web store and contains 90 capsules. According to the label each capsule contains $10 \mathrm{mg}$ of methasterone. The manufacturer recommends taking the "nutritional supplement" in cyclic periods and suggests four cycles, which vary in duration time (3-4 weeks) and daily dose (1-3 capsules). Furthermore, the manufacturer suggests pausing the methasterone cycle for one month (man) or three months (women) before beginning a new cycle.

\section{Obtaining human urine samples for the excretion study}

A human trial was set up with four young (22-29 years old) healthy male volunteers to investigate the urinary metabolites of methasterone. A written informed consent was given prior to enrollment by each volunteer, and the study protocol followed the ethical principles of medical research established at the Declaration of Helsinki was approved by the ethical committee of the University Hospital of the Federal University of Rio de Janeiro (protocol No. 020/00). Each volunteer collected a blank urine sample $24 \mathrm{~h}$ prior to taking the capsule and a sample a few minutes before taking one methasterone capsule in the morning. All the blank urine samples obtained from the four volunteers were added to the unique bottle. The four volunteers also collected postadministration urine samples separately for 30 days. On day 1 , the postadministration urine samples were collected by the volunteers and kept in separate bottles. On days 2 to $15,20,25$ and 30, only the first morning urine was collected. All urine samples were stored at $-20^{\circ} \mathrm{C}$ until analysis and were analyzed separately. The volunteers completed the study and have not reported any health problems after taking the capsule. 
Preparation of the "nutritional supplement" capsule for GC-MS analysis

The content of one M-Drol capsule was put in a glass tube, and $10 \mathrm{~mL}$ of methanol was added. After shaking for $5 \mathrm{~min}$ and centrifuging for $5 \mathrm{~min}$ at $1512 \mathrm{~g}$, the methanolic fraction was isolated. Three aliquots of $10 \mu \mathrm{L}$ each were taken from this fraction. The first aliquot was directly injected onto the GC-MS (see Instrumental analysis by GC-MS sub-section). The second aliquot was submitted to mild nitrogen flow at $40^{\circ} \mathrm{C}$ for evaporating the methanol, generating the capsule residue (see Preparation of all capsule and urine residues for instrumental analysis sub-section). The third aliquot was spiked with $10 \mu \mathrm{g}$ of methasterone standard and submitted to mild nitrogen flow at $40{ }^{\circ} \mathrm{C}$ for evaporating the methanol, generating a residue containing compounds from the capsule and the methasterone standard (see Preparation of all capsule and urine residues for instrumental analysis sub-section).

Preparation of urine samples to analyze the phase I metabolites obtained from the glucuronide fraction

Urine samples obtained through the excretion study were prepared by the screening procedure for anabolic steroids and $\beta_{2}$-agonists, as previously described ${ }^{10}$ with the following modifications. ${ }^{20}$ For GC and UPLC analysis, aliquots of 2 and $4 \mathrm{~mL}$ of urine samples were used, respectively. These aliquots were spiked with $1.0 \mu \mathrm{g}$ of IS. The pH was adjusted to 7.0 with $750 \mu \mathrm{L}$ of an aqueous buffer solution containing $\mathrm{Na}_{2} \mathrm{HPO}_{4} / \mathrm{NaH}_{2} \mathrm{PO}_{4}$ $0.8 \mathrm{M}$ and mixed briefly on a vortex mixer. Next, 1000 units of $\beta$-glucuronidase from $E$. coli were added, and hydrolysis was performed for $1 \mathrm{~h}$ at $50{ }^{\circ} \mathrm{C}$. The mixture was alkalinized to $\mathrm{pH} 10$ with $500 \mu \mathrm{L}$ of aqueous buffer solution containing $\mathrm{K}_{2} \mathrm{CO}_{3} / \mathrm{KHCO}_{3} 20 \% \mathrm{~m} \mathrm{~m}^{-1}$ each. The analytes were extracted with $5 \mathrm{~mL}$ of TBME. The mixture was stirred for $5 \mathrm{~min}$ and centrifuged at $1512 \mathrm{~g}$ for $5 \mathrm{~min}$. The ethereal phase was transferred to a fresh glass tube and evaporated to dryness under a mild nitrogen flow at $40{ }^{\circ} \mathrm{C}$, generating the urine residues (see Preparation of all capsule and urine residues for instrumental analysis sub-section).

Preparation of urine samples to analyze the free fraction, the sulfate and Cys/NAC phase II metabolites

The urine samples selected for the analysis were prepared according to the procedure performed by Gómez et al..$^{21}$ for the direct detection of sulfate, Cys and NAC conjugates and free phase I metabolites. The aliquots containing $4 \mathrm{~mL}$ of urine were spiked with $1.0 \mu \mathrm{g}$ of IS and alkalinized to $\mathrm{pH} 10$ with $500 \mu \mathrm{L}$ of aqueous buffer solution containing $\mathrm{K}_{2} \mathrm{CO}_{3} / \mathrm{KHCO}_{3} 20 \% \mathrm{~m} \mathrm{~m}^{-1}$ each. The analytes were extracted with $5 \mathrm{~mL}$ of TBME. The mixture was stirred for $5 \mathrm{~min}$ and centrifuged at $1512 \mathrm{~g}$ for $5 \mathrm{~min}$. The ethereal phase was transferred to a fresh glass tube and evaporated to dryness under a mild nitrogen flow at $40{ }^{\circ} \mathrm{C}$, generating the urine residues (see Preparation of all capsule and urine residues for instrumental analysis sub-section).

Preparation of all capsule and urine residues for instrumental analysis

The capsule and urine residues generated in the last steps were prepared for instrumental analysis. For GC-MS analysis, the capsule and urine residues were dried in a vacuum oven at room temperature for $30 \mathrm{~min}$ and then derivatized with $100 \mu \mathrm{L}$ of MSTFA/ $\mathrm{NH}_{4} \mathrm{I} / 2$-mercaptoethanol (1000:2:6, v:m:v) by heating for $20 \mathrm{~min}$ at $60{ }^{\circ} \mathrm{C} .{ }^{22}$ For UPLC-MS/MS analysis, the urine residues were resuspended in $1 \mathrm{~mL}$ of a mixture of $\mathrm{H}_{2} \mathrm{O}: \mathrm{ACN}(1: 1, \mathrm{v}: \mathrm{v})$ with $0.1 \% \mathrm{v} \mathrm{v}^{-1}$ formic acid.

\section{Instrumental analysis by GC-MS}

The GC-MS analysis was performed on an Agilent 6890 GC coupled to Agilent 5973 MS (Palo Alto, California, USA). GC parameters were as follows: the carrier gas was helium at $0.6 \mathrm{~mL} \mathrm{~min}^{-1}$ in constant flow mode; capillary column $100 \%$ polymethylsiloxane (Ultra- $1^{\circledR}$, $17 \mathrm{~m} \times 0.2 \mathrm{~mm} \times 0.11 \mu \mathrm{m} ; \mathrm{J} \& \mathrm{~W}$ Scientific, Agilent Technologies Inc., California, USA); injector temperature: $300{ }^{\circ} \mathrm{C}$; injection volume: $3 \mu \mathrm{L}$; injection mode: pulsed split with 50 psig pressure for $0.80 \mathrm{~min}$; split ratio $1: 10$. The GC oven temperature was programmed to increase from 140 to $180{ }^{\circ} \mathrm{C}$ (rate of $40{ }^{\circ} \mathrm{C} \mathrm{min}^{-1}$ ), and then was set to increase from 180 to $230{ }^{\circ} \mathrm{C}$ (rate of $3{ }^{\circ} \mathrm{C} \mathrm{min}-1$ ), and finally, to increase from 230 to $300{ }^{\circ} \mathrm{C}$ (rate of $40{ }^{\circ} \mathrm{C} \mathrm{min}-1$ ), followed by a hold for $3 \mathrm{~min}$. The MS parameters were as follows: electron ionization at $70 \mathrm{eV}$; ion source temperature: $230{ }^{\circ} \mathrm{C}$; quadrupole temperature: $150{ }^{\circ} \mathrm{C}$; transfer line temperature: $300{ }^{\circ} \mathrm{C}$. Mass spectra were acquired in full scan modes in the range $m / z, 50-750$. The data were analyzed on Enhanced ChemStation software (Agilent Technologies, New Castle, USA).

Instrumental analysis by UPLC-MS/MS

The UPLC-MS/MS analyses were performed on a Waters Acquity UPLC I-Class interfaced to a Waters Xevo TQ MS equipped with an electrospray (ESI) interface (Milford, Massachusetts, USA). 
The UPLC Acquity UPLC HSS $\mathrm{C}_{18}$ column $(50 \mathrm{~mm} \times 2.1 \mathrm{~mm} \times 1.8 \mu \mathrm{m})$ was kept inside the oven at $30{ }^{\circ} \mathrm{C}$. The mobile phases $\mathrm{A}$ and $\mathrm{B}$ were water and acetonitrile, respectively, both with $0.1 \% \mathrm{v} \mathrm{v}^{-1}$ formic acid. The flow rate was $0.3 \mathrm{~mL} \mathrm{~min}^{-1}$. In gradient mode, the $\mathrm{B}$ mobile phase was kept at $10 \%$ until the retention time $\left(t_{R}\right)$ of $0.31 \mathrm{~min}$, and then increased linearly until $60 \%$ (10.37 min) and until 90\% (10.90 min), and then kept constant until $11.43 \mathrm{~min}$, followed by a return to the initial condition at $11.69 \mathrm{~min}$, which was maintained until $13.00 \mathrm{~min}$.

The following MS/MS parameters were used. For the experiments in positive ionization mode, the spray voltage was $3.0 \mathrm{kV}$ and the declustering potential was $25 \mathrm{~V}$. The source and desolvation temperatures were 150 and $450^{\circ} \mathrm{C}$, respectively. The desolvation gas flow was $700 \mathrm{~L} \mathrm{~h}^{-1}$. All the samples prepared for UPLC-MS/MS analysis were submitted to several mass spectrometry experiments, which are described below.

\section{Precursor ion scan experiment for nontarget detection of steroids}

This experiment was carried out using high collision energy to find precursor ions of $\mathrm{m} / \mathrm{z} 77,91$ and 105 in positive ionization mode. ${ }^{23}$ The screening was done from $\mathrm{m} / \mathrm{z}, 250$ to 450 . The collision energies were $50 \mathrm{~V}$ for precursor ions of $m / z 77$ and $45 \mathrm{~V}$ for precursor ions of $\mathrm{m} / \mathrm{z} 91$ and 105 .

Product ion scan experiment for detection of methasterone and its metabolites

The experiments were performed to define the product ions of $m / z 319,333,335$ and 337, which are the pseudo-molecular ions $[\mathrm{M}+\mathrm{H}]^{+}$of methasterone and its probable metabolites. The collision energy was $25 \mathrm{~V}$, and screening was carried out from $m / z 50$ to 350 .

Precursor ion scan experiment for detection of sulfate conjugates

This experiment was performed to determine the precursor ions of $\mathrm{m} / \mathrm{z}, 97$ (hydrogen sulfate anion) in negative ionization mode ${ }^{21}$ using a collision energy of $30 \mathrm{~V}$. The screening was carried out from $\mathrm{m} / \mathrm{z} 300$ to 500 .

\section{Experiments of neutral loss and precursor ion scan for} detection of Cys and NAC conjugates

The experiments were performed as previously reported by Pozo et al..$^{24}$ The experiments of neutral loss monitored the neutral losses of 121 and $163 \mathrm{Da}$ (molecular mass of Cys and NAC, respectively) using a collision energy of $20 \mathrm{~V}$. The precursor ion scan experiments were carried out for $\mathrm{m} / z, 122$ and 164 (pseudo-molecular ions $[\mathrm{M}+\mathrm{H}]^{+}$of
Cys and NAC, respectively) using a collision energy of $10 \mathrm{~V}$. In both experiments, the screening was performed from $m / z, 300$ to 550 .

\section{Results and Discussion}

"Nutritional supplement" analysis by GC-MS

The total ion chromatogram of the M-Drol underivatized methanolic solution showed a single peak at $13.0 \mathrm{~min}$, which was from methasterone. The chromatogram obtained after derivatization showed an abundant peak at $a t_{R}$ of $15.3 \mathrm{~min}$, whose intensity increased after adding the methasterone standard, and a minor peak at $14.9 \mathrm{~min}$ (Figure S1, Supplementary Information (SI) section). Their mass spectra are equivalent to the methasterone standard trimethylsilyl (TMS) derivative mass spectrum. Taking into account their abundances, $\mathrm{t}_{\mathrm{R}}$ and that methasterone is a 17-hydroxy-2 $\alpha$-methyl- $5 \alpha$-androstane steroid, it is possible to say that the peaks at 14.9 and $15.3 \mathrm{~min}$ are from the 3 -enol and 2-enol TMS derivatives, respectively. ${ }^{25,26}$ Other steroids were not detected in the capsule.

Detection of methasterone and its phase I metabolites from glucuronide fractions using GC-MS

Taking into account the expected phase I metabolism routes for methasterone, diagnostic fragments were selected to find their respective metabolite TMS derivatives. The search for metabolites with intact D rings was performed by using their most abundant fragment, $m / z, 143,{ }^{27}$ while the C16-hydroxylated metabolites were searched by their abundant fragments, $\mathrm{m} / \mathrm{z} 218$ and $231 .{ }^{28}$ Screening for C12-hydroxylated metabolites was accomplished through the fragments $\mathrm{m} / \mathrm{z} 170^{15,17}$ and 185 to find metabolites with intact D rings. By extension, the possible 12,16-dihydroxylated metabolites were investigated through the fragments $m / z, 258$ and 273. Screening for the 16-oxo metabolites was performed through the abundant fragment $\mathrm{m} / z, 244 . .^{28}$ The possible 12-hydroxy-16-oxo metabolites were investigated through the fragments $\mathrm{m} / \mathrm{z}, 256$ and 271. As a hydroxylation at $\mathrm{C} 18$ could lead to the loss of $-\mathrm{CH}_{2} \mathrm{OH}$ group, the generation of the compounds 18-nor-13(14)-ene, 18-nor-17,17-dimethyl-13(14)-ene and/or 18-nor-17-methyl,17-hydroxymethyl-13(14)-ene was considered. ${ }^{12}$ Thus, the screening for the 18 -nor17-methyl,17-hydroxymethyl-13(14)-ene metabolite was performed by the abundant fragments $\mathrm{m} / \mathrm{z}, 133,236^{11}$ and $303 .{ }^{17}$ By extension, the 18-nor-13(14)-ene metabolite and 18-nor-17,17-dimethyl-13(14)-ene metabolite were screened by the possible abundant fragments $m / z, 222$ and 
148 , respectively, which are both analogues of $m / z 236$, and $\mathrm{m} / \mathrm{z} 289$ and 215, respectively, which are both analogues of $\mathrm{m} / \mathrm{z}$ 303. Furthermore, possible 18-nor compounds containing a 12-hydroxy group were evaluated using the cited ions $(\mathrm{m} / \mathrm{z}, 133,236,303,222,148,289$ and $215)$ plus $88 \mathrm{Da}$, which is related to the -OTMS group. Additionally, the 6-hydroxy metabolites were screened using $m / z, 312$ (A ring with 3-oxo group) and $m / z, 314$ (A ring with 3-hydroxy group). All these screening strategies are summarized on Table 1. Moreover, screening for C18-hydroxylated metabolites was performed through fragments $m / z$ 103, related to the cation $\left[\mathrm{CH}_{2} \mathrm{OTMS}\right]^{+}$and $[\mathrm{M}-103]^{+} .{ }^{28}$ Screening for 13 -carboxy metabolites that could be originated from C18-hydroxylated metabolite oxidation $^{8}$ was performed through the abundant fragment $\mathrm{m} / \mathrm{z} 117$ (carboxy-TMS cation). Unfortunately, only the screening for $\mathrm{m} / \mathrm{z}, 143,218$ and 231 was able to find metabolites. Therefore, only methasterone and nine metabolites were detected.

The methasterone and phase I metabolite concentrations in human urine from the $1^{\text {st }}$ to the $30^{\text {th }}$ day after the administration of methasterone were evaluated. First, an abundant and diagnostic fragment ion was chosen for methasterone and for each phase I metabolite TMS derivative. The peak area of this fragment was integrated in each chromatogram, as well as the peak area of the $m / z 301$ ion, generated from the IS. For each phase I metabolite TMS derivative and urine sample, the relative analyte/IS peak area was calculated. As the IS concentration was known, it was possible to calculate the concentration of methasterone and each metabolite in each urine sample. Therefore, the average concentration and the standard deviation for all metabolites at each day after administration were estimated using the data obtained from four people. On the first day, the volunteers collected several urine samples, and only the most concentrated urine sample for each metabolite and volunteer was used in the calculation.

The methasterone 2-enol-TMS derivative eluted at 15.7 min giving the main fragments $m / z 143,462$ (molecular ion), 447 (loss of methyl radical) and 372 (loss of TMSOH, whose molecular mass is $90 \mathrm{Da}$, from the molecular ion) as seen at Figure 2. These fragments were also detected at $10.0 \mathrm{~min}$ in the postadministration urine samples but were not detected in the capsule and blank urine samples, suggesting a TMS derivative from a methasterone diastereoisomer produced by human metabolism, M1 (Figure 3). It was thought that M1 would be a methasterone epimer at $\mathrm{C} 17$, as predicted by Fragkaki et al. ${ }^{12}$ However, we suggest that a $\mathrm{C} 17$ epimerization only would not be sufficient to shift the $t_{R}$ from 15.7 to 10.0 min. By comparing the methasterone and M1 TMS derivative mass spectra (Figures 2 and 3, respectively),

Table 1. Diagnostic fragments used to screen methasterone metabolite TMS derivatives. The values are expressed in $\mathrm{m} / \mathrm{z}$ and are related to the fragmentations F1-F7 shown in the picture

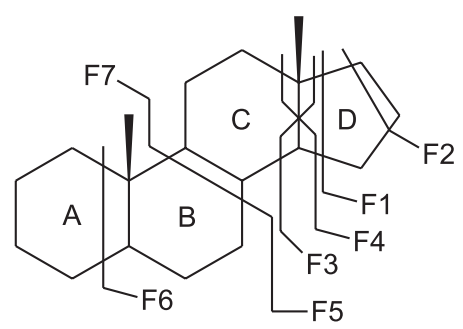

\begin{tabular}{|c|c|c|}
\hline \multicolumn{3}{|c|}{ Fragments related to metabolites with modifications at $\mathrm{C}$ and/or $\mathrm{D}$ rings } \\
\hline & & 12-Hydroxy metabolites \\
\hline D ring intact & $\mathrm{F} 1=143$ & $\mathrm{~F} 4=170,185$ \\
\hline 16-Hydroxy & $\mathrm{F} 1=231, \mathrm{~F} 2=218$ & $\mathrm{~F} 4=258,273$ \\
\hline 16-Oxо & $\mathrm{F} 3=244$ & $\mathrm{~F} 4=256,271$ \\
\hline \multicolumn{3}{|c|}{ Fragments related to metabolites that could be generated after the loss of $-\mathrm{CH}_{2} \mathrm{OH}$ group } \\
\hline & $\mathrm{C} 12$ position intact & 12-Hydroxy metabolites \\
\hline 18-Nor-17-methyl-17-hydroxymethyl-13(14)-ene & $133, \mathrm{~F} 5=236, \mathrm{~F} 6=303$ & $221, \mathrm{~F} 5=324, \mathrm{~F} 6=391$ \\
\hline 18-Nor-13(14)-ene & $133, \mathrm{~F} 5=222, \mathrm{~F} 6=289$ & $221, \mathrm{~F} 5=310, \mathrm{~F} 6=377$ \\
\hline 18-Nor-17,17-dimethyl-13(14)-ene & $133, \mathrm{~F} 5=148, \mathrm{~F} 6=215$ & $221, \mathrm{~F} 5=236, \mathrm{~F} 6=303$ \\
\hline \multicolumn{3}{|c|}{ Fragments related to metabolites with modifications at $\mathrm{A}$ and/or B rings } \\
\hline & A ring intact & 3-Hydroxy metabolites \\
\hline 6-Hydroxy & $\mathrm{F} 7=312$ & $\mathrm{~F} 7=314$ \\
\hline
\end{tabular}


it can be seen that the latter shows a molecular ion that is significantly less abundant than in the former. This fact could suggest that M1 is a $5 \beta$ steroid, which is less stable than $5 \alpha$ due to steric reasons. To confirm this hypothesis, we analyzed $5 \alpha / \beta$ epimer standards of $5 \xi$-androstadione, a 3 -oxo steroid of methasterone, on our GC instrument. The results showed that the epimer $5 \beta$ eluted $3.1 \mathrm{~min}$ before the $5 \alpha$ (Rt of 9.8 and $12.9 \mathrm{~min}$, respectively) and it also had a less abundant molecular ion than the $5 \alpha$ (Figure S2, SI section). Their TMS derivatives were proposed according to Parr et al. ${ }^{26}$ Taking into account the $\mathrm{t}_{\mathrm{R}}$ and the fragmentation profiles, we considered the hypothesis of epimerization at C5, generating M1 (17ß-hydroxy$2 \alpha, 17 \alpha$-dimethyl-5 $\beta$-androstan-3-one) to be valid, and
M1 is a new methasterone metabolite candidate because it was previously unreported, to the best of our knowledge. It is important to highlight that M1 is slightly more polar than methasterone due to its TMS derivative $t_{R}$, which corroborates the importance of epimerization reactions for human metabolism and urinary excretion.

The M2 and M3 TMS derivatives were found at 13.3 and $13.8 \mathrm{~min}$, respectively, and have similar mass spectra (Figures S3 and S4, SI section) and characteristic fragments of a 3-hydroxy metabolite TMS derivative. M2, a 3 $\alpha$-hydroxy metabolite already detected in vivo ${ }^{13,17}$ and in vitro, ${ }^{14,15}$ is routinely monitored at our laboratory. M3 has been found only in vitro. ${ }^{15}$ It is possible to assume that $\mathrm{M} 3$ is the $3 \beta$ epimer due to its abundance

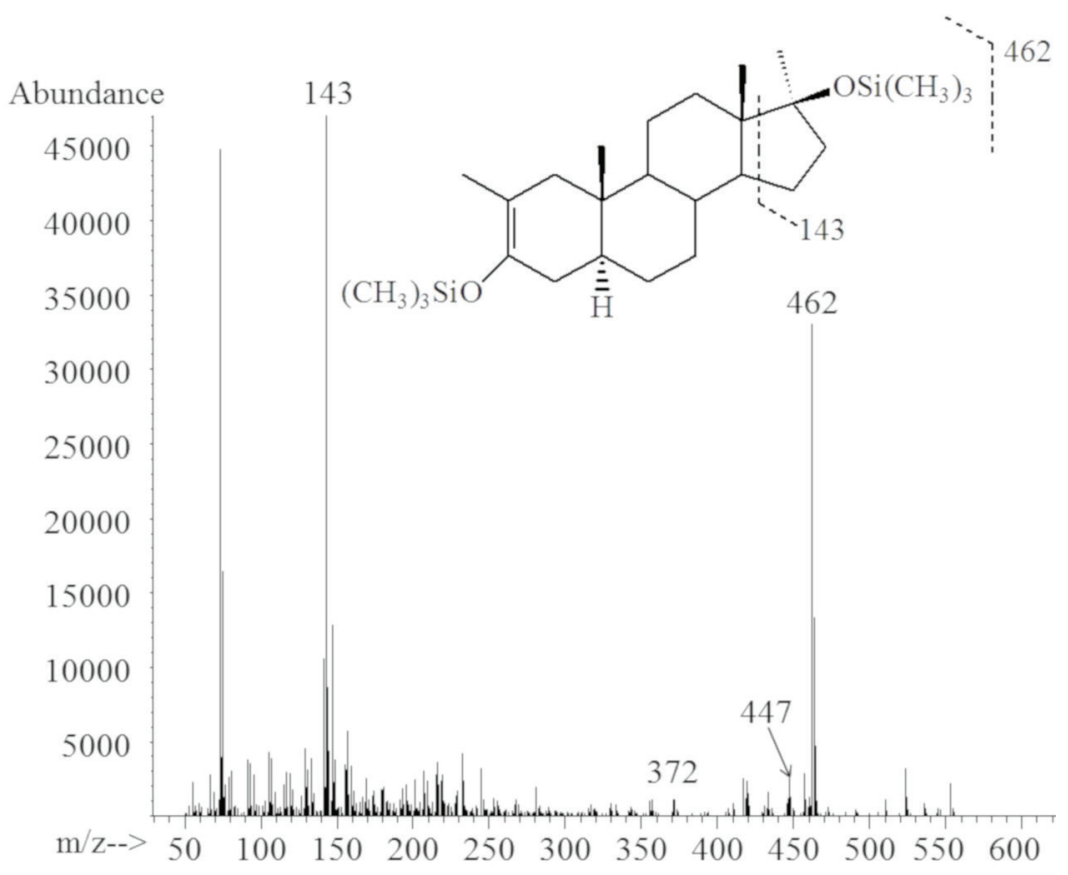

Figure 2. Postadministration urine mass spectrum at $15.7 \mathrm{~min}$ (where the methasterone 2-enol-TMS derivative eluted) and its proposed fragmentation pathways.

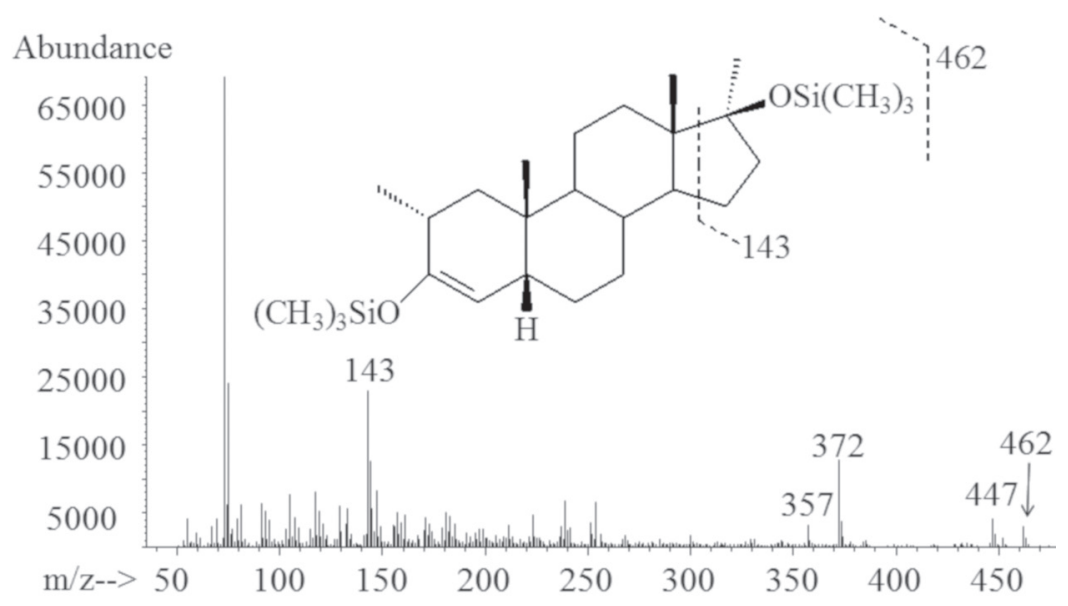

Figure 3. Postadministration urine mass spectrum at 10.0 min (where the M1 3-enol-TMS derivative eluted) and its proposed fragmentation pathways. 
and retention time. According to Gauthier et al. ${ }^{15}$ the $3 \alpha$ epimer is more abundant than the $3 \beta$ epimer. M2 and M3 present maximum estimated concentrations of 140 and $20 \mathrm{ng} \mathrm{mL}^{-1}$, respectively. A comparison of the M2 and M3 retention times with the data obtained by Fragkaki et al. ${ }^{29}$ using $3 \alpha$ and $3 \beta$ isomers of $5 \alpha$-androsta-3,17 $\alpha$-diol TMS derivatives contributes to this hypothesis because the $3 \alpha$ epimer elutes before the $3 \beta$ epimer. Thus, M2 could be $2 \alpha, 17 \alpha$-dimethyl- $5 \alpha$-androsta- $3 \alpha, 17 \beta$-diol, and M3 could be $2 \alpha, 17 \alpha$-dimethyl-5 $\alpha$-androsta-3 $\beta, 17 \beta$-diol.

The M4 and M5 TMS derivatives were found at 17.2 and $16.8 \mathrm{~min}$, respectively, and have similar mass spectra (Figures S5 and S6, SI section) and characteristic fragments as the 2 $\beta, 3 \alpha$-diol metabolite TMS derivative reported by Gauthier et al. ${ }^{15}$ These authors found two 2,3-diol metabolites and proposed, through chemical synthesis and nuclear magnetic resonance, that the major isomer would be a $2 \beta, 3 \alpha$-diol metabolite. Furthermore, this compound was also found in humans ${ }^{13,17}$ and in vitro. ${ }^{14}$ As M4 and M5 have respective maximum estimated concentrations of 50 and $10 \mathrm{ng} \mathrm{mL}^{-1}$, it is possible to suppose that M4 is the $2 \beta, 3 \alpha$-diol isomer $(2 \alpha, 17 \alpha$-dimethyl- $5 \alpha$-androsta$2 \beta, 3 \alpha, 17 \beta$-triol) and M5 is an M4 diastereoisomer ( $2 \xi, 17 \alpha$-dimethyl-5 $\alpha$-androsta- $2 \xi, 3 \xi, 17 \beta$-triol).

The M6 TMS derivative was detected at $19.0 \mathrm{~min}$ and has similar mass spectrum (Figure S7, SI section) and characteristic fragments as a $16 \xi$-hydroxy metabolite TMS derivative that has already been reported. ${ }^{15,17}$ Therefore, M6 appears to be $16 \xi, 17 \beta$-dihydroxy-2 $\alpha, 17 \alpha$-dimethyl$5 \alpha$-androstan-3-one.

The M7 TMS derivative was detected at $18.1 \mathrm{~min}$ and has similar mass spectrum (Figure S8, SI section) and characteristic fragments as a 3,16-dihydroxy metabolite TMS derivative that has already been published. ${ }^{15}$ Because M7 could be generated from M2 or M3 after oxidation at $\mathrm{C} 16$, it is more probable that $\mathrm{M} 2$ is the M7 precursor, because it is more abundant than $\mathrm{M} 3$, as already mentioned. Therefore, M7 is probably $2 \alpha, 17 \alpha$-dimethyl- $5 \alpha$-androsta$3 \alpha, 16 \xi, 17 \beta$-triol.

The M8 TMS derivative was detected at $19.3 \mathrm{~min}$ and has similar mass spectrum (Figure S9, SI section) and characteristic fragments as a 2,3,16-trihydroxy metabolite TMS derivative that has already been found..$^{15,16}$ Due to its high abundance, it is probable that M8 has M4 as its precursor. M8 is probably $2 \alpha, 17 \alpha$-dimethyl-5 $\alpha$-androsta$2 \beta, 3 \alpha, 16 \xi, 17 \beta$-tetrol.

The M9 TMS derivative was found at $19.6 \mathrm{~min}$ and gives the fragments $\mathrm{m} / \mathrm{z} 218,231,623$ (loss of methyl group), 548 (loss of TMSOH) and the molecular ion $\mathrm{m} / \mathrm{z} 638$ (Figure 4), which indicates a methasterone TMS derivative with two extra-OTMS groups. The ions $\mathrm{m} / z 218$ and 231 show that one extra-OTMS group is at C16, while the minor fragment, $\mathrm{m} / \mathrm{z} 508$, enables the determination of the other-OTMS group at C2 (Figure S10, SI section). This diagnostic fragment probably arises from a cleavage at the A ring, as proposed by Gauthier et al. ${ }^{15}$ for the fragment $\mathrm{m} / \mathrm{z}, 420$ generated from $2 \alpha, 17 \alpha$-dimethyl-5 $\alpha$-androsta$2 \beta, 3 \alpha, 17 \beta$-triol (our M4 metabolite) TMS derivative. Lootens et al. ${ }^{16}$ found a metabolite whose TMS derivative has fragments $\mathrm{m} / \mathrm{z} 638,218$ and 231, but they did not determine the extra-OTMS group position. Moreover, the mass spectrum shown by these authors does not match with our spectrum because theirs shows an abundant molecular ion, and the fragment $m / z 231$ is more abundant than 218 . Besides of these authors, Geldof et al. ${ }^{14}$ reported in 2016 the metabolite $2 \beta, 16 \xi, 17 \beta$-trihydroxy-2 $\alpha, 17 \alpha$-dimethyl$5 \alpha$-androstan-3-one, which has molecular mass $638 \mathrm{Da}$ and hydroxy groups at C2 and C16 such as M9. As these authors have not shown the mass spectrum of its metabolite, it is impossible to say if M9 corresponds to such molecule. Therefore, M9 is probably $2 \xi, 16 \xi, 17 \beta$-trihydroxy$2 \alpha, 17 \alpha$-dimethyl-5 $\alpha$-androstan-3-one and is generated

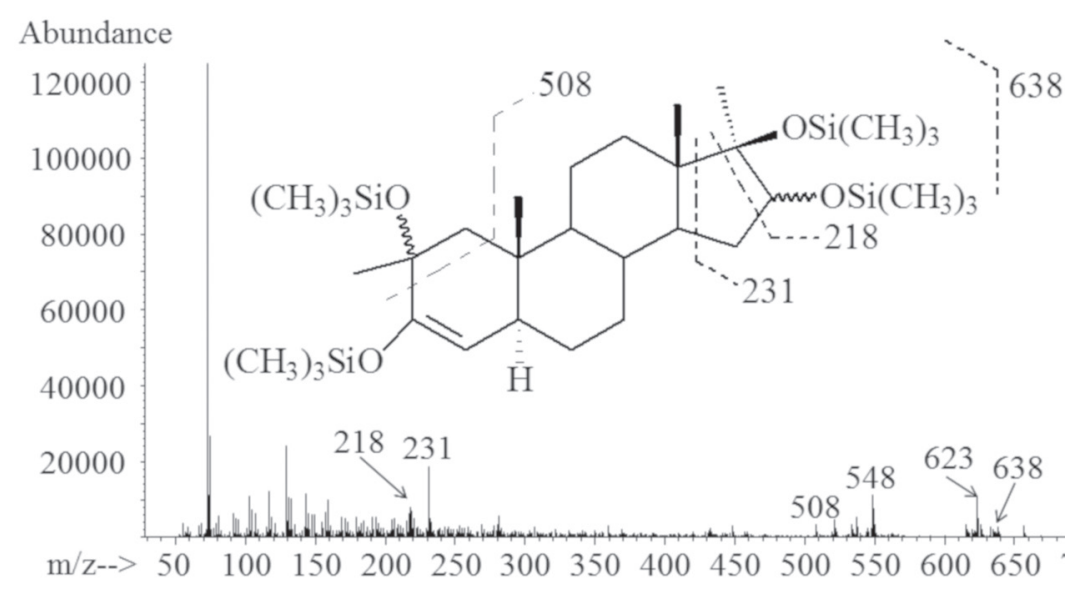

Figure 4. Postadministration urine mass spectrum at $19.6 \mathrm{~min}$ (where the M9 TMS derivative eluted) and its proposed fragmentation pathways. 
from M6, taking into account that M9 is less abundant than M6, and it is possible to be a new metabolite.

The proposed chemical structure of candidate metabolites M1 to M9 and their suggested metabolism routes are shown in Figure 5.

The estimated concentration of methasterone and its metabolites M1 to M9 was evaluated for 30 days.
Methasterone and its metabolites, except M1, began to be excreted a few hours after drug administration. As the metabolites were detected during nine days or less, only a portion of the full data (from the $1^{\text {st }}$ to the $10^{\text {th }}$ day) is shown in Figure 6 . From the $10^{\text {th }}$ day to the end of the study, no metabolites were detected. After drug administration, the most abundant compounds were methasterone and

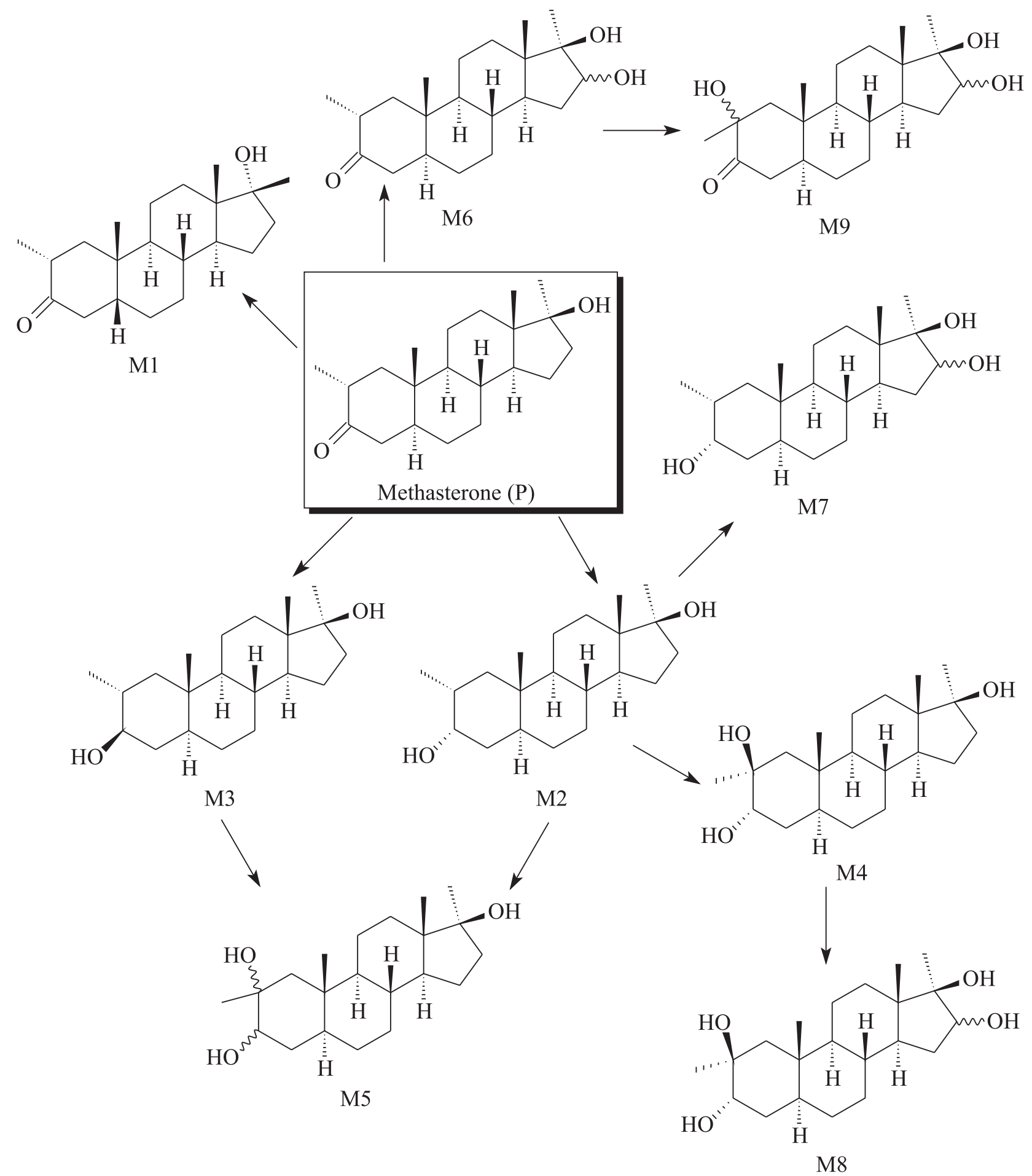

Figure 5. Proposed chemical structures of M1 to M9 methasterone metabolites and suggested pathways for their biotransformation in humans. 
M2, followed by M8 and M4. Methasterone and M2 are monitored by anti-doping laboratories and were detected until the $3^{\text {rd }}$ and $9^{\text {th }}$ days, respectively. However, they were detected for 11 and 13 days, respectively, through GC coupled to high-resolution mass spectrometry (GC-HRMS). ${ }^{13}$ According to our results, M1 and M4 were detected until the $9^{\text {th }}$ day as well. Interestingly, M1 was excreted by only two volunteers and began to be detected on the last day in which methasterone was found, that is, on the $3^{\text {rd }}$ day. M6 and M8 were excreted until the $6^{\text {th }}$ and $8^{\text {th }}$ days, respectively. Among the minor metabolites, M3, M5 and M7 were detected for only two days, while M9 was detected for nine days. Despite the low number of volunteers and the low sensitivity of GC-MS, it is possible to deduce that M4, M8 and M9 are good metabolites for monitoring in addition to $\mathrm{M} 2$, because they seem to be excreted for a period that is as long as M2, but this needs to be verified by GC-HRMS and/or GC-MS/MS. Moreover, it is important to include other metabolites in routine doping analysis due to usual biological variations.
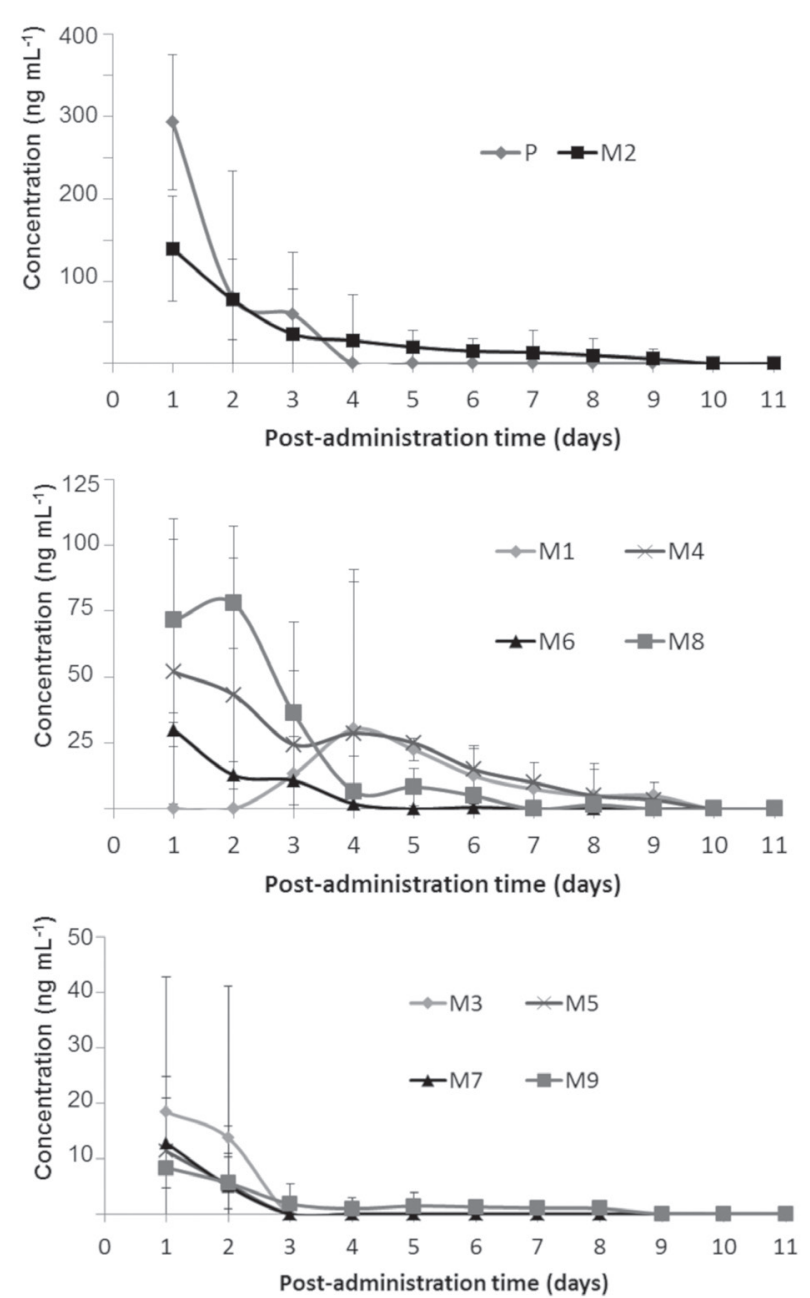

Figure 6. Urinary excretion profile of methasterone $(\mathrm{P})$ and its metabolites M1 to M9. The volunteers took the methasterone on day 1 .
Detecting methasterone and its phase I metabolites from free fractions using GC-MS

The possibility of methasterone and its nine metabolites to be excreted in free form was also investigated. The urine samples selected for the analysis were prepared and analyzed by GC-MS without previous hydrolysis by $\beta$-glucuronidase, but methasterone and its metabolites were not found.

Screening for methasterone and its phase I metabolites from glucuronide fractions using UPLC-MS/MS

Through the experiments that scanned precursor ions of $\mathrm{m} / z, 77,91$ and 105 , only methasterone was found, which occurred at 9.9 min using the fragment $\mathrm{m} / \mathrm{z}$ 105. The ions $\mathrm{m} / \mathrm{z} 77$ (phenyl ion), 91 (tropylium ion) and 105 (methyl tropylium ion) are useful to detect steroids because they can be generated from the complete fragmentation of either the $\mathrm{B}$ or the $\mathrm{C}$ ring. ${ }^{23}$ The product ion spectrum of $m / z 319$ (pseudo-molecular ion $[\mathrm{M}+\mathrm{H}]^{+}$of methasterone) is shown in Figure S11 (SI section). In addition to the $\mathrm{m} / \mathrm{z} 319$ ion, it is important to mention $\mathrm{m} / \mathrm{z} 301$, which is related to a neutral water loss from the pseudo-molecular ion.

Using $\mathrm{m} / \mathrm{z}, 319,333,335$ and 337 product ion scan experiments, it was possible to detect only one metabolite from methasterone. Its pseudo-molecular ion $[\mathrm{M}+\mathrm{H}]^{+}$ has a mass of $335 \mathrm{Da}$ and was found at $8.0 \mathrm{~min}$ through the $m / z, 335$ product ion scan experiment, whose mass spectrum is shown in Figure S12 (SI section). In addition to the $m / z 335$ ion, it is important to mention the fragments $\mathrm{m} / \mathrm{z} 317$ and 299, which originated from two consecutive neutral water losses. The data indicate that methasterone was hydroxylated at a saturated carbon. Taking into account the structures of M1 to M9, which were previously found by GC-MS, it is possible to say that such metabolite found by UPLC-MS/MS is M6.

\section{Screening for phase II metabolites from urine samples using UPLC-MS/MS}

The structural condition for the conjugation of drugs with sulfate is the presence of nucleophilic groups such as hydroxy and amine; therefore, it is fundamental to investigate whether methasterone and its metabolites are also excreted as sulfate conjugates. A valid strategy to find sulfate conjugates is through UPLC-MS/MS using an $\mathrm{m} / \mathrm{z} 97$ precursor ion scan in negative mode due to the generation of a hydrogen sulfate anion from the metabolite because the mass of this anion is $97 \mathrm{Da}^{21}$ This experiment was performed using postadministration samples and blank urine, 
but their chromatograms did not show abundant peaks in the postadministration samples and were absent in the blank urine. Therefore, methasterone and its metabolites were not significantly excreted as sulfate conjugates by the volunteers.

In addition to the sulfate conjugates, the presence of Cys and/or NAC conjugates was evaluated by UPLC-MS/MS. Cys and NAC conjugates have a similar behavior in positive ionization mode at low collision energy. ${ }^{24}$ They can lose the neutral amino acid (whose molecular mass is $121 \mathrm{Da}$ for Cys and $163 \mathrm{Da}$ for NAC), generating the pseudo-molecular ion $[\mathrm{M}+\mathrm{H}]^{+}$of the free drug. They can also lose the neutral drug, generating the pseudo-molecular ion $[\mathrm{M}+\mathrm{H}]^{+}$of the amino acid (122 Da for Cys and $164 \mathrm{Da}$ for NAC). The experiments of neutral loss were done for 121 and $163 \mathrm{Da}$ using postadministration samples and blank urine. Similarly, the experiments of the protonated amino acid precursor ion scan were done for $\mathrm{m} / \mathrm{z} 122$ and 164. However, the obtained chromatograms did not show peaks in the postadministration samples, and peaks were absent in the blank urine samples. Thus, methasterone and its metabolites were not significantly excreted as Cys and/or NAC conjugates.

\section{Conclusions}

Nine phase I metabolites were characterized in the glucuronide fraction of the urine from the excretion studies. One of them was not yet reported: $17 \beta$-hydroxy$2 \alpha, 17 \alpha$-dimethyl-5 $\beta$-androstan-3-one, obtained from the epimerization at C5. Phase I metabolites were not found in free form. Sulfate, cysteine and $\mathrm{N}$-acetylcysteine were not detected either. It is important to include some additional long-term methasterone metabolites, such as M4, M8 and/ or M9, into routine anti-doping analyses as soon as possible. The reason for is their long detection window, which is as long as the currently monitored metabolite, whose production may be affected by variation in human metabolism.

\section{Supplementary Information}

Supplementary information (Figures S1-S12) is available free of charge at http://jbcs.sbq.org.br as PDF file.

\section{Acknowledgments}

The authors appreciate the support from $\mathrm{CNPq}$ and FUJB.

\section{References}

1. Sjöqvist, F.; Garle, M.; Rane, A.; Lancet 2008, 371, 1872.
2. Bowers, L. D.; Clark, R. V.; Shackleton, C. H.; Steroids 2009 , 74, 285.

3. Sekera, M. H.; Ahrens, B. D.; Chang, Y. C.; Starcevic, B.; Georgakopoulos, C.; Catlin, D. H.; Rapid Commun. Mass Spectrom. 2005, 19, 781.

4. WADA 2006 Prohibited List available at https://www.wadaama.org/sites/default/files/resources/files/WADA_Prohibited_ List_2006_EN.pdf, accessed in November 2017.

5. WADA 2018 Prohibited List available at https://www.wadaama.org/sites/default/files/prohibited_list_2018_en.pdf, accessed in September 2018.

6. Shah, N. L.; Zacharias, I.; Khettry, U.; Afdhal, N.; Gordon, F. D.; Clin. Gastroenterol. Hepatol. 2008, 6, 255.

7. Nasr, J.; Ahmad, J.; Dig. Dis. Sci. 2009, 54, 1144.

8. Williams, D. A. In Foye's Principles of Medicinal Chemistry, $6^{\text {th }}$ ed.; Lemke, T. L.; Williams, D. A.; Roche, V. F.; Zito, S. W., eds.; Lippincott Williams \& Wilkins: Philadelphia, USA, 2008.

9. Masse, R.; Goudreault, D.; J. Steroid Biochem. Mol. Biol. 1992, 42, 399.

10. Schänzer, W.; Donike, M.; Anal. Chim. Acta 1993, 275, 23.

11. Schänzer, W.; Geyer, H.; Fusshöller, G.; Halatcheva, N.; Kohler, M.; Parr, M. K.; Guddat, S.; Thomas, A.; Thevis, M.; Rapid Commun. Mass Spectrom. 2006, 20, 2252.

12. Fragkaki, A. G.; Angelis, Y. S.; Tsantili-Kakoulidou, A.; Koupparis, M.; Georgakopoulos, C.; J. Steroid Biochem. Mol. Biol. 2009, 115, 44.

13. Bylina, D. V.; Gryn, S. V.; Tkachuk, A. A.; Methods Objects Chem. Anal. 2012, 7, 87.

14. Geldof, L.; Lootens, L.; Polet, M.; Eichner, D.; Campbell, T.; Nair, V.; Botrè, F.; Meuleman, P.; Leroux-Roels, G.; Deventer, K.; van Eenoo, P.; Biomed. Chromatogr. 2014, 28, 974; Geldof, L.; Tudela, E.; Lootens, L.; van Lysebeth, J.; Meuleman, P.; Leroux-Roels, G.; van Eenoo, P.; Deventer, K.; Biomed. Chromatogr. 2016, 30, 1202.

15. Gauthier, J.; Goudreault, D.; Poirier, D.; Ayotte, C.; Steroids 2009, 74, 306.

16. Lootens, L.; Meuleman, P.; Leroux-Roels, G.; van Eenoo, P.; J. Steroid Biochem. Mol. Biol. 2011, 127, 374.

17. Zhang, J.; Lu, J.; Wu, Y.; Wang, X.; Xu, Y.; Zhang, Y.; Wang, Y.; Int. J. Mol. Sci. 2016, 17, 1628.

18. Tudela, E.; Deventer, K.; Geldof, L.; van Eenoo, P.; Drug Test. Anal. 2015, 7, 95.

19. Weththasinghe, S. A.; Waller, C. C.; Fam, H. L.; Stevenson, B. J.; Cawley, A. T.; McLeod, M. D.; Drug Test. Anal. 2018, 10, 330.

20. Pereira, H. M. G.; Padilha, M. C.; Bento, R. M. A.; Cunha, T. P.; Lascas, N. A. G.; Aquino-Neto, F. R.; TrAC, Trends Anal. Chem. 2008, 27, 648.

21. Gómez, C.; Pozo, O. J.; Fabregat, A.; Marcos, J.; Deventer, K.; van Eenoo, P.; Segura, J.; Ventura, R.; Drug Test. Anal. 2012, 4,775 . 
22. Pereira, H. M. G.; Marques, M. A. S.; Cardoso, J. N.; AquinoNeto, F. R.; Quim. Nova 2002, 25, 1096.

23. Pozo, O. J.; Deventer, K.; van Eenoo, P.; Delbeke, F. T.; Anal. Chem. 2008, 80, 1709.

24. Pozo, O. J.; Gómez, C.; Marcos, J.; Segura, J.; Ventura, R.; Drug Test. Anal. 2012, 4, 786.

25. de Boer, D.; de Jong, E. G.; Maes, R. A.; van Rossum, J. M.; J. Steroid Biochem. Mol. Biol. 1992, 42, 411.

26. Parr, M. K.; Zapp, J.; Becker, M.; Opfermann, G.; Bartz, U.; Schänzer, W.; Steroids 2007, 72, 545.
27. Borges, C. R.; Taccogno, J.; Crouch, D. J.; Le, L.; Truong, T. N.; Int. J. Mass Spectrom. 2005, 247, 48.

28. Fragkaki, A. G.; Angelis, Y. S.; Tsantili-Kakoulidou, A.; Koupparis, M.; Georgakopoulos, C.; Int. J. Mass Spectrom. 2009, 285, 58.

29. Fragkaki, A. G.; Tsantili-Kakoulidou, A.; Angelis, Y. S.; Koupparis, M.; Georgakopoulos, C.; J. Chromatogr. A 2009, $1216,8404$.

Submitted: November 17, 2018

Published online: January 22, 2019 